\title{
MRI Versus Ultrasound In Diagnosis Of Meniscal Tear In Knee Joint
}

\author{
* Hossam Adel Mohamed Mostafa ${ }^{1}$, Ahmed Mohamad Abou Elfotuh ${ }^{1}$, Mohamad Mobarak \\ Alsakka ${ }^{1}$ \\ Department of Radiodiagnosis, Faculty of Medicine, Al-Azhar University \\ *Corresponding author: Hossam Adel Mohamed,e-mail:hosamadelmohammed@gmail.com, mobile: -01014200044
}

\begin{abstract}
Background: the menisci of the knee are complex structures with various important functions within the knee. Loss of the menisci leads to a significantly increased risk of developing degenerative changes in the long term.
\end{abstract}

Purpose: this study aimed to investigate the role of Ultrasound (US) in diagnosis of meniscus tears as compared to Magnetic Resonance Imaging (MRI)

Patients and Methods: this study included 50 patients, presented by pain, swelling, stiffness/limitation of movement or a history of acute/chronic knee trauma, in the duration between November 2017 and July 2018. The research was carried on the Radiology Department, Al Azhar General Hospital, Zagazig, Sharkia Governorate, Ministry of Health. All patients underwent musculoskeletal ultrasound in different position then underwent magnetic resonance imaging included different pulse sequences and scanning planes.

Results: this study included 50 patients, $31(62 \%)$ males and $19(38 \%)$ females. The patient's age ranged between 10 and 67 years with a mean of $37.65 \pm 10.24 S \mathrm{D}$. Male patient's age ranged between 10 and 67 years with a mean of $36.35 \pm 11.03 \mathrm{SD}$, while female patient's age ranged between 29 and 55 years with a mean of $40 \pm 8.34 S \mathrm{D}$.

Conclusion: high resolution ultrasonography had high accuracy in detecting presence of tears in both the medial and lateral menisci. MRI is more sensitive in detection and determines types of tears than US.

Keywords: MRI Knee, ultrasound, menisci

\section{INTRODUCTION}

Meniscal tears are the most widely recognized pathology of the knee with a mean yearly occurrence of 66 for every 100000 . Verifiably it was trusted that the menisci filled no utilitarian need ${ }^{(\mathbf{1})}$. Menisci are critical for load transmission, stun ingestion, and joint adjustment (2). Knee wounds are normal, particularly when participating in games people. Wounds to delicate tissues, for example, tendons, ligament and ligaments are normally experienced. Harm deep down additionally can happen. The knee joint is a compound sort of synovial joint and because of the absence of hard help, solidness of the joint is very reliant on its supporting ligamentous structures, and in this manner wounds of tendons and menisci are amazingly normal ${ }^{(3)}$. Exact and convenient determination of a meniscal tear is basic for diminishing dreariness and arranging treatment. It is entrenched that meniscal harm inclines the nearby articular ligament to expanded hub and sheer pressure, bringing about early degenerative osteoarthritis the pervasiveness of asymptomatic tears, which ordinarily are even tears, increments with age. The consolidation of clinical data and the rejection of an elective reason can permit certain conclusion of a symptomatic meniscal tear ${ }^{(4)}$. Precise finding of meniscal tear relies on imaging. Knee arthrography has been to a great extent supplanted by Magnetic reverberation imaging (MRI). MRI is presently the analytic strategy for decision in assessment of menisci. The precision of MRI in diagnosing meniscal tears has been accounted for to be higher than $90 \%$. In any case, the utilization of MRI isn't just costly, yet additionally has a few confinements including metallic inserts, claustrophobia, tolerant movement curios, and a long examination time. Attractive reverberation imaging (MRI) isn't in every case promptly open. It has been proposed to utilize ultrasonography for the investigation of the menisci of the knee and especially to analyze tears since 1989. Ultrasonography is a less expensive, quicker and more accessible technique than MRI. The affectability and particularity of a few investigations on the 
estimation of ultrasonography in the finding of meniscal tears have changed enormously, so the utilization of sonography in this setting stays questionable ${ }^{(5)}$. MRI of the knee has been observed to be exceptionally exact in the conclusion of meniscal tears. In past investigations, the affectability of MRI in the identification of meniscal tears has been accounted for to be somewhere in the range of $80 \%$ and $100 \%$. Kelly et al. announced $94 \%$ precision of MRI contrasted and arthroscopy in the recognition of meniscal tears. Mink et al. announced $92 \%$ precision for MRI contrasted and arthroscopy ${ }^{(4)}$.

While, attractive reverberation imaging (MRI) is regularly considered the "best quality level" indicative imaging methodology for identification of meniscal irregularities, it is related with misdiagnosis in as high as $47 \%$ of cases, is exorbitant, and isn't promptly accessible to countless. Ultrasonographic examination of the knee has been accounted for to be a successful demonstrative device for this reason with the possibility to conquer a considerable lot of the deficiencies of MRI ${ }^{(6)}$.

\section{Aim of the work}

The aim of this research was to compare between the accuracy of Ultrasound and MRI in diagnosis and evaluation of meniscal tear.

\section{Patients and methods}

This study included 50 patients, presented by pain, swelling, stiffness/limitation of move-ment or a history of acute/chronic knee trauma, in the duration between November 2017 and July 2018. The research was carried on the Radiology Department, Al Azhar General Hospital, Zagazig , Sharkia Governorate, Ministry of Health. This study was approved by the Ethics Board of AlAzhar University.

All the patients underwent musculo-skeletal ultrasound in different position then underwent magnetic resonance imaging including different pulse sequences and scanning planes. Inclusion criteria included:

1. History of knee trauma.

2. Patients presented by chronic knee pain, swelling or stiffness.

\section{Exclusion criteria included:}

General contraindications for MR imaging (e.g. cardiac pace maker)

Claustrophobic patients.

\section{Patient Preparation for ultrasound:}

No specific patient preparation was required Technique for ultrasound

Sonographic exams (GE logic and Toshiba oxario) were performed with 7.5 to $12 \mathrm{MHz}$ probes in prone and supine positions through the anterior, lateral and posterior approaches using static and dynamic techniques.

Most of the imaging was done in the longitudinal plane. In the static technique, the anterior horns of the menisci were imaged in supine position with the knee in full extension and 30-45 degrees of flexion. Then the probe was moved to sides to visualize the bodies of the menisci. Afterwards the patients were placed prone and posterior horns were imaged with the knee in extension first then 45 degrees flexion. In dynamic imaging, the knee was subjected to mild internal and external Varus stresses to allow better imaging of the menisci using movements.

\section{Patient Preparation for MRI:}

The patients were questioned about history of intracranial surgical clips, cardiac pace-makers, cochlear implants and metallic objects in the body before the MR examination. No specific patient preparation or sedation was required. The clinical details and any prior imaging diagnostic result were available. The patients were examined in supine position.

\section{Technique for MRI:}

MRI was performed for all patients on (1.5 T) Phillips Achieva MRI machine.

1-Patients were placed supine with the knee extended and slightly externally rotated (10$15^{\circ}$ ) in an extremity coil to optimize the signal to noise ratio.

2-Images were obtained mainly in both the sagittal and coronal planes, where sagittal images were done with the knee externally rotated to permit imaging in the plane of the ACL. Axial images were also scanned to study the supporting ligaments around the knee.

3-Routine MRI sequences were used including turbo spin echo sagittal proton density; T1 and T2 weighted images as well as coronal STIR \& axial T2 weighted images. Additional sequences were sometime used as sagittal STIR, coronal T1 or T2 weighted images. These were obtained using a field of view of 16$20 \mathrm{~cm}$, slice thickness of 3-5 mm, and a matrix of $352 \times 320$. A skip of $(0-20 \%$ of slice thickness) was used between imaging sections.

Data analysis and Statistical data display: 
To obtain diagnostic values, we measured sensitivity, specificity, positive and negative predictive values of the analyzed data using commercially available PC-based software pack-age (SPSS)

\section{Results}

The result included 50 patients, $31(62 \%)$ males and $19(38 \%)$ females as table shows below

Table 1: average age of included patients in relation to their sex

\begin{tabular}{l|l|l|l|l}
\hline Age & Min. & Max. & Mean & S.D. \\
\hline \hline Male & 10 & 67 & 36.35 & 11.03 \\
Female & 29 & 55 & 40.00 & 8.34 \\
\hline All & 10 & 67 & 37.65 & 10.24 \\
\hline \hline
\end{tabular}

A total of 62 meniscal lesions in this study population were included. Of these 24 (38.7\%) were in the left knee, while $38(61.3 \%)$ were in the right knee. The distribution of these lesions according to the site in the meniscus is plotted in Table.

Table 2: distribution of site of the included meniscal lesions

\begin{tabular}{l|l|l}
\hline & Count & Percentage \\
\hline \hline PHMM & 32 & $51.61 \%$ \\
AHMM & 6 & $9.68 \%$ \\
PHLM & 12 & $19.35 \%$ \\
AHLM & 12 & $19.35 \%$ \\
\hline \hline
\end{tabular}

The final diagnosis of the 62 meniscal lesions according to MRI examination was meniscal degeneration in $44(71 \%)$ lesions, and meniscal tear in 18 (29\%) lesions (Table 3).

Table 3: MRI diagnosis of meniscal lesions in correlation with their site

\begin{tabular}{|c|c|c|c|c|c|c|c|c|}
\hline & \multicolumn{2}{|c|}{ PHMM } & \multicolumn{2}{|c|}{ AHMM } & \multicolumn{2}{|c|}{ PHLM } & \multicolumn{2}{|c|}{ AHLM } \\
\hline & $\mathrm{N}$ & $\%$ & $\mathrm{~N}$ & $\%$ & $\mathrm{~N}$ & $\%$ & $\mathrm{~N}$ & $\%$ \\
\hline Normal & 0 & $0.00 \%$ & 0 & $0.00 \%$ & 0 & $0.00 \%$ & 0 & $0.00 \%$ \\
\hline Degeneration & 22 & $35.48 \%$ & 4 & $6.45 \%$ & 10 & $16.13 \%$ & 8 & $12.90 \%$ \\
\hline Tear & 10 & $16.13 \%$ & 2 & $3.23 \%$ & 2 & $3.23 \%$ & 4 & $6.45 \%$ \\
\hline
\end{tabular}

The distribution MRI diagnosed according to types of tear was demonstrated in the following table:

Table 4: MRI diagnosis of meniscal lesions according to the types of tears

\begin{tabular}{l|l|l|l|l}
\hline \multicolumn{2}{l|}{} & N & \% of lesion group & \% of total \\
\hline Degeneration & Grade 2 & 44 & $100.00 \%$ & $70.97 \%$ \\
\hline \multirow{3}{*}{ Tear } & Horizontal & 14 & $77.78 \%$ & $22.58 \%$ \\
& Bucket Handle & 2 & $11.11 \%$ & $3.23 \%$ \\
& Flap & 2 & $11.11 \%$ & $3.23 \%$ \\
\hline \hline
\end{tabular}

On the other hand, out of 62 lesions, ultrasound missed 6 (9.7\%) lesions, while it diagnosed 30 (48.4\%) lesions as meniscal degeneration, and $26(42.1 \%)$ lesions as meniscal tears. 26 (42\%) lesions were detected in the posterior horn of medial meniscus. It should be noted that, in this study, all meniscal lesions that were missed by ultrasound were located in the posterior horn of medial meniscus. Table 5 Error! Reference source not found.. 
Table 5: ultrasound diagnosis of meniscal lesions in correlation with their site

\begin{tabular}{|c|c|c|c|c|c|c|c|c|}
\hline & $\begin{array}{l}\text { PHMM } \\
\mathrm{N}\end{array}$ & $\%$ & $\begin{array}{l}\text { AHMM } \\
\mathrm{N}\end{array}$ & $\%$ & $\begin{array}{l}\text { PHLM } \\
\mathrm{N}\end{array}$ & $\%$ & $\begin{array}{l}\text { AHLM } \\
\mathrm{N}\end{array}$ & $\%$ \\
\hline Normal & 6 & $9.68 \%$ & 0 & $0.00 \%$ & 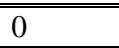 & $0.00 \%$ & 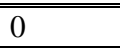 & $0.00 \%$ \\
\hline Degeneration & 4 & $22.58 \%$ & 0 & $0.00 \%$ & 8 & $12.90 \%$ & 8 & $12.90 \%$ \\
\hline Tear & 2 & $19.35 \%$ & 6 & $9.68 \%$ & 4 & $6.45 \%$ & 4 & $6.45 \%$ \\
\hline
\end{tabular}

Out of 44 degenerative meniscal lesions that were diagnosed with MRI, Ultrasound could correctly diagnose 28 lesions, while 10 lesions were diagnosed as meniscal tears, 6 degenerative lesions were missed by ultrasound.

On the other hand, out of 18 meniscal tears diagnosed with MRI, Ultrasound could correctly diagnose 16 tears, while 2 lesions were reported as degenerative lesions.

A statistically significant difference was found between the diagnostic performance of MRI and ultrasound ( $\mathrm{p}$-value $=0.003$ ).

Table 6

Table 6: comparison between MRI and Ultrasound diagnosis of meniscal lesions

\begin{tabular}{|c|c|c|c|c|c|c|}
\hline & & & $\begin{array}{l}\text { MRI } \\
\text { Normal }\end{array}$ & Degeneration & Tear & Total \\
\hline \multirow{3}{*}{ US } & Normal & $\begin{array}{l}\mathrm{N} \\
\%\end{array}$ & $\begin{array}{l}0 \\
0.00 \%\end{array}$ & $\begin{array}{l}6 \\
9.68 \%\end{array}$ & $\begin{array}{l}0 \\
0.00 \%\end{array}$ & $\begin{array}{l}6 \\
9.68 \%\end{array}$ \\
\hline & Degeneration & $\begin{array}{l}\mathrm{N} \\
\%\end{array}$ & $\begin{array}{l}0 \\
0.00 \% \\
\end{array}$ & $\begin{array}{l}28 \\
45.16 \%\end{array}$ & $\begin{array}{l}2 \\
3.23 \% \\
\end{array}$ & $\begin{array}{l}30 \\
48.39 \% \\
\end{array}$ \\
\hline & Tear & $\begin{array}{l}\mathrm{N} \\
\%\end{array}$ & $\begin{array}{l}0 \\
0.00 \%\end{array}$ & $\begin{array}{l}10 \\
16.13 \%\end{array}$ & $\begin{array}{l}16 \\
25.81 \%\end{array}$ & $\begin{array}{l}26 \\
41.94 \%\end{array}$ \\
\hline
\end{tabular}

Regarding meniscal tears, Ultrasound was consistent with MRI in $50(80.65 \%)$ lesions out of 62 . As it yielded 16 true positive and 34 true negative lesions Table. Sensitivity of Ultrasound in detecting meniscal tears was hence found to be $88.89 \%$, specificity was $77.27 \%$, while accuracy was $80.65 \%$.Error! Reference source not found.

Table 7: ultrasound versus MRI in diagnosis of meniscal tears

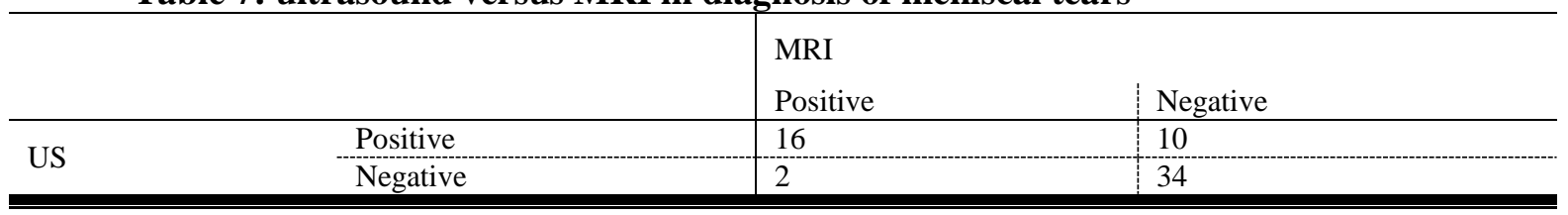

On the other hand, regarding meniscal degeneration, Ultrasound was consistent with MRI in 44 $(70.97 \%)$ lesions out of 62 ; it yielded 28 true positive and 16 true negative lesions. Table showing sensitivity of ultrasound in detecting meniscal degeneration was hence found to be $63.64 \%$, specificity was $88.89 \%$, while accuracy was $70.97 \%$ (Error! Reference source not found.).

Table 8: ultrasound versus MRI in diagnosis of meniscal degeneration

\begin{tabular}{|c|c|c|c|}
\hline & & \multicolumn{2}{|l|}{ MRI } \\
\hline & & Positive & Negative \\
\hline \multirow{2}{*}{ US } & Positive & 28 & 2 \\
\hline & Negative & 16 & 16 \\
\hline
\end{tabular}

\section{Case: 1}

\section{Ultrasound findings:}

RT knee showed linear hypoechoic streak reaching the articular surface of PHLM. Picture suggestive of PHLM tear (Fig.1). 


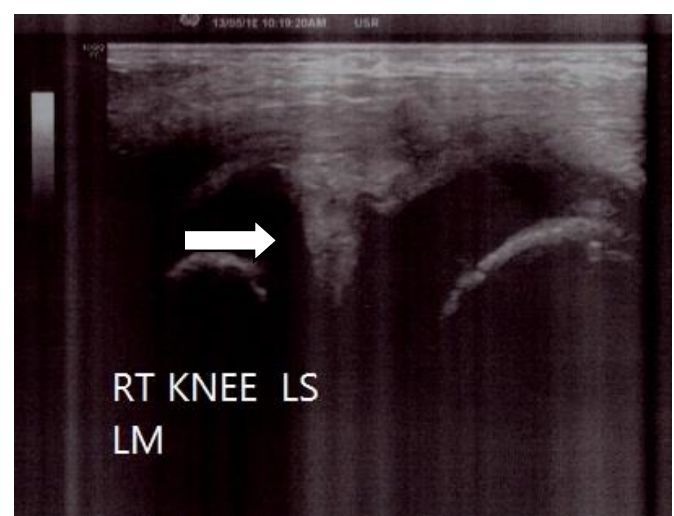

Figure 1: RT knee LS hypoechoic line reaching articular surface at PHLM

MRI findings: (Figure 1).

In MRI the PHLM show abnormal signal intensity not reached articular surface at sagittal PD w/fat picture suggestive of grade II degeneration meniscal tear

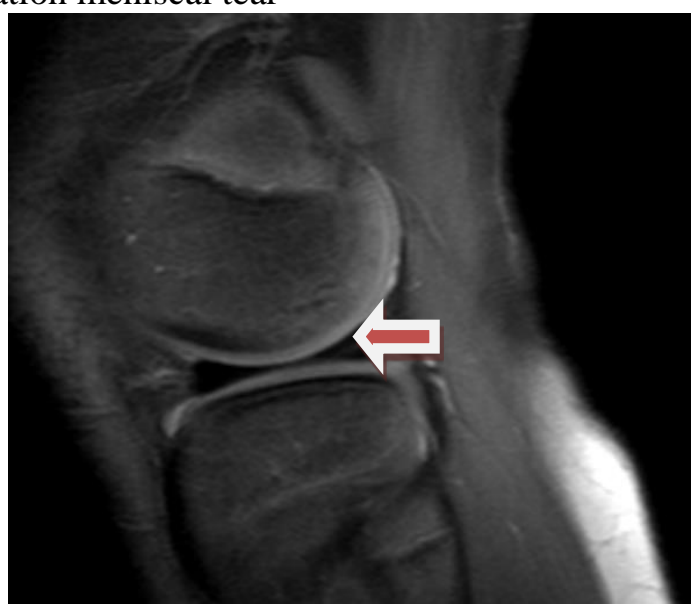

Figure 1. Sagittal PD W/fat shows abnormal signal intensity within the posterior horn of the lateral meniscus not reached the articular surface "Grade 2" (arrow)

Case 2:

Ultrasound findings :(Figure ).

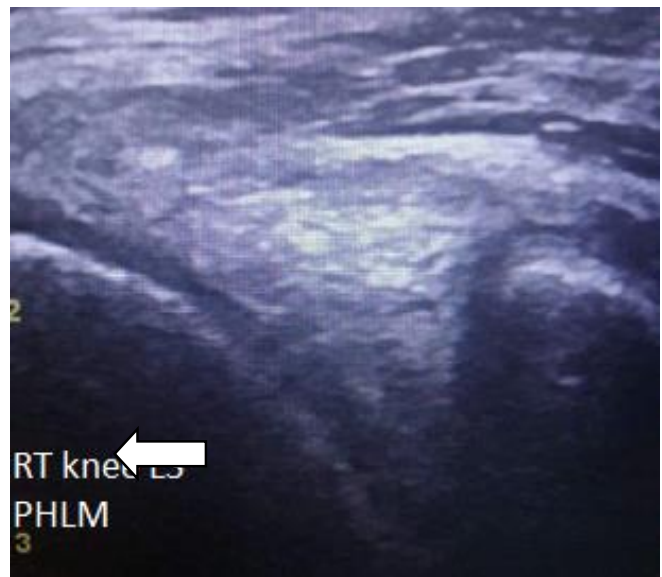

RT knee showed linear hypoechoic streak reaching the articular surface of PHLM. Picture suggestive of PHLM tear.

Figure 3: hypoechoic tear is seen in posterior horn lateral meniscus,, reached the

MRI findings: (Figure 4).

$$
\text { articular surface }
$$

In MRI the PHLM showed abnormal signal intensity reaching articular surface at sagittal PD w/fat picture suggestive of flap tear (Fish mouth tear) 


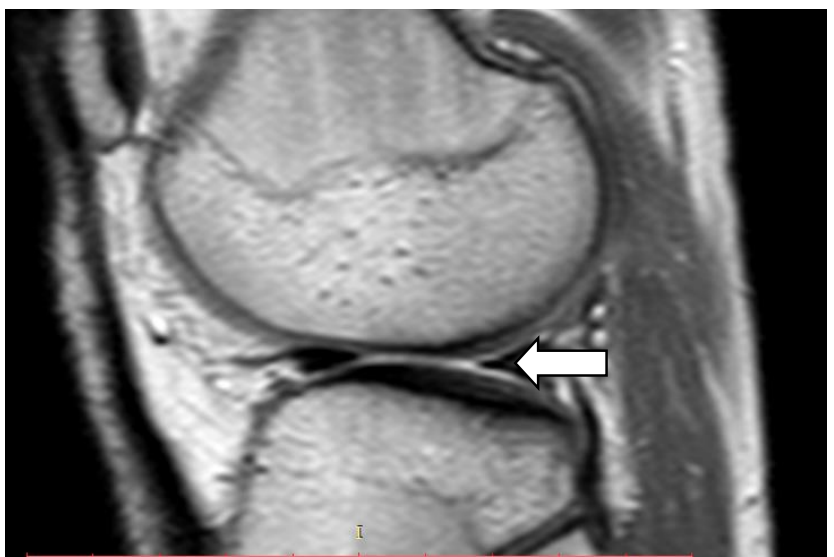

Figure 4: sagittal PD W/FAT shows abnormal signal intensity within the posterior horn of the lateral meniscus reached the articular surface flap tear (Fish mouth) (arrow).

\section{DISCUSSION}

The most common causes of knee pain and disability are tears in medial or lateral menisci. Meniscal injuries are common in both athletes and the general population.In the last decade, musculoskeletal imaging has rapidly expanded due to the imaging capabilities of magnetic resonance imaging and ultrasound. A study showed that clinical examination alone did not give adequate information to demonstrate arthroscopic examinations. In this way, imaging contemplates were quite often required. Arthroscopy was the best quality level test for meniscal sores which can be utilized both for analysis and for treatment in the meantime, anyway intrusiveness was a burden and furthermore fringe tears might be missed if the examination was not cautiously and completely led ${ }^{(7)}$ As of late, intrigue has developed whether US can be utilized as an elective test to MRI in meniscal imaging. US, as opposed to MRI was a generally accessible and an ease test and has an expanding job in the musculoskeletal applications too ${ }^{(8)}$. Anyway, one principle inconvenience of US was being broadly administrator ward and picture debasement identified with specialized elements or relics from hard and delicate tissue (9). in this study we wanted to determine whether US can be used as an alternative to MRI for detection of tears and degeneration.Regarding the distribution of the patients according to sex, in our study the percentage of males was $62 \%$ and the percentage of females was $38 \%$ in a group of 50 patients, in comparison to $78 \%$ \& $22 \%$ respectively in a group of 39 patients in the study carried out by Nasir ${ }^{(10)}$. This could be explained by the fact that males are more vulnerable to such traumatic knee injury during daily activity and sports injury, while females were more vulnerable to meniscal degeneration resulting from weight bearing due to obesity.The patient's age in our study ranged between 10 and 67 years with a mean of 37.65 \pm 10.24 SD. Taking in mind the distribution of the lesions according to the affected horn, $70.96 \%$ was the percentage of the posterior horn lesions, and $29.04 \%$ was the percentage of the anterior horn lesions, which was away different from the results reported by Nasir ${ }^{(10)}$, with a percentage of $46.2 \%$ posterior horn lesions and $53.8 \%$ anterior horn lesions. Regarding our statistical results for meniscal tears. Ultrasound was consistent with MRI in 50 $(80.65 \%)$ lesions out of 62 ; as it yielded 16 true positive and 34 true negative lesions. Sensitivity of Ultrasound in detecting meniscal tears was hence found to be $88.89 \%$, specificity was $77.27 \%$, while accuracy was $80.65 \%$.In comparison to the study done by El-Monem $\boldsymbol{e t}$ al. (11), the sensitivity of US in diagnosis of meniscal tears were $80.5 \%$, specificity was $76.9 \%$, while accuracy was $80 \%$. Results are nearly the same. Our results also are in agreement with the findings of Peterson $\boldsymbol{e t}$ al. (12) who reported a sensitivity of $86 \%$ and specificity of $83 \%$ for conventional sonography in the diagnosis of meniscal tears. In our study, it was difficult to comment on the type of tear with an ultrasonography evaluation alone. However, in MRI it can easily determine types of tears as we found horizontal tears represent $77.78 \%$ and bucket handle tear represent 11.11 $\%$ and flap tear represent $11.11 \%$ of the lesion group. Our study had a few limitations. Apart from the smaller sample size of only 50 patients, in spite of the attempt to undertake a 
blind review, the absolute blinding was difficult to achieve because of the obvious difference in image quality between the two modalities made some bias inevitable. In addition, the accuracy of MRI in the diagnosis of meniscal tears was dependent on the experience of the interpreter and his or her knowledge of the potential imaging pitfalls. Correlation with arthroscopy was not obtained as the arthroscopy with solely diagnostic purpose was not done routinely at our institution and not all patients need a therapeutic arthroscopic procedure.On other hand, ultrasonography may be used as a screening tool prior to arthroscopy in selected cases where MRI was a contraindication or is not available or if the patient was not affording. Ultrasonography showed a dynamic image of the meniscus and thus may prove useful if studied in conjunction with a proper clinical examination. It was difficult to comment on the type of tear with an ultrasonography evaluation alone.

\section{Conclusion}

High-resolution ultrasonography (US) had high accuracy in detecting presence of tears in both the medial and lateral menisci. MRI was more sensitive in detection and determine types of tears than US. At the end we recommended that if there was a patient with history of knee trauma and clinical suspicion of meniscal injuries, we recommend to start with high resolution ultrasound examination as screening tool. For negative examinations follow-up, if no improvement the second step is MRI examination to rule out meniscal injuries. For positive results although ultrasound can never replace MRI, which was the modality of choice, it was a good low-cost alternative when MRI was not available or when waiting period for MRI can cause unnecessary delay in management.

\section{References}

1-Simon C Mordecai, Nawfal Al-Hadithy, Howard $E$ Ware and Chinmay M Gupte (2014): Treatment of meniscal tears: An evidence based approach. World Journal of Orthopedics, 5(3): 233-241.
2- Sharifah M, Lee C et al.(2015) : Accuracy of MRI in the diagnosis of meniscal tears in patients with chronic ACL tears. Knee Surgery, Sports Traumatology, Arthroscopy, 23(3):826830.

3-Bhanupriya S et al. (2016): Evaluation of knee joint by ultrasound and MRI. Journal of Dental and Medical Sciences,15: 122-131.

4-Jie C et al. (2014): MR imaging-based dagnosis and classification of meniscal tears. Radiographics, 34:981-999.

5-Alizade A et al. (2012) : Knee sonography as a screening test for medial meniscus tearing in young patients. European Society of Radiology, 11: 100-109.

6-James L et al. (2014) : MRI versus ultrasonography to assess meniscal abnormalities in acute knees. The Journal of Knee Surgery, 27:319-324.

7- Ravichandra G, Aravinda M, Sajeer Mo $U$ and Vivek S (2014) : Usg and MRI correlation in the evaluation of meniscal lesionsof knee. Journal of Evolution of Medical and Dental Sciences,DOI: 10.14260/jemds/2014/3482.

8-EL Beblawy M et al. (2004): Normal and acutely torn anterior cruciate ligament of the knee at US evaluation. The Egyptian Journal of Radiology and Nuclear Medicine, 18: 325-340. 9-Najafi J, Bagheri S and Lahiji F (2006): The value of sonogra-phy with micro convex probes in diagnosing meniscal tears compared with arthroscopy. J. Ultrasound Med., 25: 593600.

10-Nasir A I(2013): The role of magnetic resonance imaging in the knee joint injuries. Int. Res. J. Medical Sci., 1(5): 1-7.

11- Abdel EL-Monem $S$ and Moanes $M$ (2012): Comparative study between high resolution ultrasound (hrus) and MRI in diagnosis of meniscal and cruciate ligaments injury of the knee. Med. J. Cairo Univ., 80(2):233-242.

12- Peterson LJ and Rasmussen O S (2009): Ultrasound as a diagnostic method in suspected meniscal lesions of the knee-a prospective single blind study of 52 patients. The Knee, 16: 191-195. 